\title{
BMJ open Those who can, teach. Assessing medical students' perception of a finals revision programme delivered by foundation and core trainees: a cross-sectional study
}

\author{
Guy Mole, ${ }^{1}$ Liam Gillespie $^{2}$
}

To cite: Mole G, Gillespie L. Those who can, teach. Assessing medical students' perception of a finals revision programme delivered by foundation and core trainees: a cross-sectional study. BMJ Open 2012;2:e001544. doi:10.1136/bmjopen-2012001544

- Prepublication history and additional material for this paper are available online. To view these files please visit the journal online (http://dx.doi.org/10.1136/ bmjopen-2012-001544).

Received 23 May 2012 Revised 31 October 2012 Accepted 1 November 2012

This final article is available for use under the terms of the Creative Commons Attribution Non-Commercial 2.0 Licence; see http://bmjopen.bmj.com

${ }^{1}$ Vascular Surgery, Royal Sussex County Hospital, Brighton, UK

${ }^{2}$ Colorectal Surgery, Royal Sussex County Hospital, Brighton, UK

Correspondence to Dr Guy Francis Asa Mole; G.Mole1@uni.bsms.ac.uk

\section{ABSTRACT}

Objective: To determine whether medical students perceive a foundation and core trainee finals revision programme as useful.

Design: Questionnaires were used to assess students' perception of the teaching programme.

Setting: A medical school in the South of England.

Participants: There were 106 final year medical students in the cohort.

Results: The teaching scored well in all facets analysed and scored best on enthusiasm, interactivity and communication.

Conclusions: We provide a framework for how foundation and core trainees could provide a very well received teaching programme benefiting medical students, the doctors and the medical school.

\section{INTRODUCTION}

The word doctor originates from the Latin root docere, 'to teach', and doctors spend the entirety of their careers as both the teachers and the students. ${ }^{1}$ Teaching is integral to developing as a doctor, ${ }^{2}$ as the 17 th Century French moralist Joseph Joubert famously asserted 'to teach is to learn twice' and teaching is emphasised as a fundamental skill in the GMC document 'Good Medical Practice'. ${ }^{3}$ Though self-learning via access to published material is of undoubted importance, medicine is an apprenticeship and knowledge is largely obtained and refined through discussion with colleagues; both seniors and peers. Recently there has been an increasing interest in the role of peers and near-peers (colleagues of only limited seniority) in undergraduate medical education, resulting in many peer-reviewed publications. $^{4-9}$ As a result, peer-assisted and near-peer tutoring has been established as a useful adjunct to structured teaching from senior faculty, and has been shown to

\section{ARTICLE SUMMARY}

\section{Article focus}

- To determine whether medical students perceive a finals revision teaching programme given by foundation and core trainees as useful.

- Which facets of teaching were rated highly.

- Should foundation and core trainees be utilised for teaching in an undergraduate curriculum.

Key messages

- The teaching scored well in all categories analysed.

- Students felt that their knowledge afterwards was better than before.

- The foundation and core trainees were rated higher on enthusiasm and communication than on knowledge.

Strengths and limitations of this study

- A 16 lecture series with multiple simulation suite and ward teaching sessions meant there was a large amount of data collected.

- Data collected from on-going questionnaires was very similar across all lectures showing a consistently highly rated programme; however, comparison with end-of-programme questionnaires was invalid due to duplicate questionnaires within the sample.

- Students were aware that this programme was put on in the doctors spare time and may have been inclined to score it more favourably.

improve exam performance when used as a supplementary source of information. ${ }^{10-12}$

Near peers have been highlighted as a potentially valuable teaching resource; it has also been suggested that routinely giving intermediate trainees responsibility for teaching aspects of the undergraduate curriculum may be of benefit for both teacher and student. ${ }^{8}$ Yet the role of foundation and core trainees in teaching medical students, with the exception of the role of anatomy demonstrator, has traditionally been somewhat more 
informal. This teaching typically consists of opportunistic moments in the clinical setting, or brief lectures given on an ad hoc basis, usually selected as a subject of interest for the teacher. Very rarely do foundation and core trainees provide a structured curriculum for the benefit of the student. Little work has been done to assess the contribution that a near-peer educator may be able to make to different aspects of the medical curriculum. In particular, there is a paucity of research into which learning environment a near-peer tutor may be best placed, and how valuable their teaching might be as a core component of the curriculum rather than a supplementary one.

The current study attempts to assess students' perception of a formal, structured, foundation and core trainee-led finals revision programme. The study asks final-year students to score the programme on several criteria including knowledge before and afterwards. The programme was designed to provide an equivalent for each facet of teaching provided by the medical school, specifically; didactic teaching, bedside teaching and clinical skills teaching.

The current study attempts, in short, to determine medical students' perception of a structured teaching programme for finals delivered by foundation and core trainees.

\section{METHODS}

The programme was developed as a comprehensive guide to both the written and clinical finals examinations at the local medical school. The programme syllabus was designed in collaboration with faculty at the school, and addressed key topics within the undergraduate final year curriculum, with a particular emphasis on those subject areas that students typically found problematic.

A 16 week revision course was compiled, covering all key topics relevant to the part one final examinations and culminating in a 10-station 'mock' Observed Structural Clinical Examination (OSCE). Because the programme coincided with final-year regional placements, a programme coordinator in each of the six regional centres attended by the students was appointed (following informal interview) to oversee the organisation for that trust. The authors served as programme coordinators for the University Teaching Hospital while also providing advice and assistance to the programme coordinators at the other centres.

The formal programme offered by the medical school consisted of one consultant-led didactic session per week at each of the regional centres over the 16 weeks, covering core topics for finals. The medical school also provided a number of clinical skills suite sessions over the 16 weeks, in addition to both ad hoc and structured bedside teaching. This was mirrored by the teaching programme which consisted of didactic and practical sessions, which aimed to cover, in appropriate detail, all material relevant to one core topic or specialty. One evening of lectures, covering core learning objectives from the curriculum was followed by a series of small group, ward-based sessions or simulator sessions in the clinical skills suite, to consolidate the information given in the lecture. The practical session also served to provide an informal environment in which to ask questions, in addition to OSCE-style practice in each field. Key revision notes, guides to OSCE-style examination and information from each lecture were made available online via the school intranet following the completion of each topic.

The 'mock' OSCE was composed of 10, 7 min stations selected to evenly represent the curriculum and was delivered to all students over 3 days from the main hospital education centre. Each student was then provided with both informal feedback and the mark sheets from their examination thus this exercise doubled as a teaching OSCE. ${ }^{13}$

The course lectures, clinical skills sessions, bedside teaching sessions, online resources and OSCE stations were designed, delivered and audited exclusively by a small group of foundation year-1 doctors. The programme was, however, delivered by a range of volunteers, with 53 of the 79 foundation year-1 doctors employed by the trust contributing to the programme at the University Hospital site, in addition to a small number of foundation year-2 doctors and core trainees.

Ethical approval was not sought from the local body, Brighton \& Sussex Medical School Research Governance and Ethics Committee (BSMS RGEC). The voluntary junior-led programme was offered in addition to the formal programme and it was made clear to all attendees that the programme was being delivered without formal oversight as to the accuracy of the course content. As such this is classified as teaching audit which does not require ethical approval. Furthermore, teaching by all doctors is an almost ubiquitous feature of undergraduate medicine, and when viewed from this perspective the study could be considered observational.

\section{On-going assessment}

Each week an anonymous feedback questionnaire was given after the lecture session, in which students were asked to rate 11 aspects of the teaching session on a fivepoint rating scale between very poor and very good. The components assessed were: structure of the session, breadth of topic, depth of topic, use of visual material, enthusiasm of teacher, interactivity with audience, communication skills, timekeeping, knowledge prior to session, knowledge following session and an overall impression. This questionnaire was designed to assess internal consistency within the programme, and to ensure that all volunteers were teaching to a comparable standard.

\section{End of programme assessment}

This consisted of an anonymous questionnaire provided at the end of the programme. There was a five-point 
global score for junior-led didactic and clinical teaching, in which students were asked to rate their impression of the programme as a whole, between very poor and very good. This questionnaire was handed out after the last lecture and also after the 'mock' OSCE.

Three sections consisted of detailed assessment of didactic teaching, ward-based teaching and clinical skillssuite teaching at the regional centre attended. Each teaching method was assessed with respect to the following components of the teacher:

- Knowledge base-whether the teacher's knowledge base was sufficient to teach at the level required and whether the teachers own framework for understanding was accessible to the student.

- Approachability-whether the teacher provided a suitably nurturing learning environment to enable students to feel able to ask questions.

- Ability to answer questions-whether answers provided in response to questions were appropriate and accessible.

- Relevance to finals-how well the student felt the material covered the finals curriculum.

- Relevance to practice-how useful the information provided would be in day to day practice.

- Amount learned-how much overall the student felt their own knowledge had improved as a result of the teaching.

The final section of the questionnaire asked the students to indicate which method of teaching and which teacher they preferred for: Preparation for finals, Preparation for practice and Learning of core knowledge.

\section{RESULTS}

In total, 231 On-going Assessments from 12 lectures were filled in at an average of 19 per lecture. More students attended each lecture but feedback was only collected at the Teaching Hospital. Only 12 were included as some lectures were excluded due to not being based on a medical school curriculum topic. These were the introductory talk, MTAS talk, 'mock' OSCE talk (explaining how the OSCE would run, not coaching the students on OSCE skills) and tidy up session.

Of a year of 106 students, 94 undertook the mock OSCE.

In total, 158 End of Programme questionnaires were completed which was more than the total in the year of 106. This was because questionnaires were handed out at the last talk and after the 'mock' OSCE showing that some were completed twice. As a result, an unknown proportion of the completed end-of-programme questionnaires were duplicates making all analyses of results from these questionnaires invalid.

\section{On-going assessment questionnaire analysis}

The On-going Assessment forms from the lecture programme were collated so that each of the 11 categories could be evaluated for the lecture series as a whole. This
Table 1 The mean score that students gave to different facets of the teaching provided by foundation and core trainees, with the standard deviation.

\begin{tabular}{lll}
\hline & Mean & SD \\
\hline Structure & 4.7 & 0.5 \\
Level & 4.7 & 0.6 \\
Knowledge & 4.6 & 0.6 \\
Visual & 4.5 & 0.7 \\
Enthusiasm & 4.8 & 0.4 \\
Interactivity & 4.8 & 0.5 \\
Communication & 4.8 & 0.5 \\
Timekeeping & 4.6 & 0.7 \\
Knowledge before & 3.3 & 1.1 \\
Knowledge after & 4.4 & 0.7 \\
Overall & 4.6 & 0.5 \\
\hline
\end{tabular}

showed that the lecture series scored well in all categories with over $90 \%$ of scores being either 4 (good) or 5 (very good). In addition the overall scores showed that of 231, 141 questionnaires scored the lecture as 5 and 80 as 4 , leaving only 10 questionnaires scoring a lecture as less than good. Data are summarised in table 1 .

The scores given for knowledge before and knowledge after were analysed using a Mann-Whitney test which was significant ( $p$ value of $<0.001$ ), indicating that students felt their knowledge had improved after the session.

\section{DISCUSSION}

The feedback analysed confirms the results of previous studies, indicating that near-peer teaching is perceived as a valuable resource by students. ${ }^{6} 14-17$

It is important to note that the course was designed and overseen by a very small group of self-selected foundation doctors. Only two foundation doctors were involved in designing the curriculum and a third with the mock OSCE. However, the programme was delivered by a substantially greater number, 19 foundation year-1 doctors contributed to the lecture series, and over two-thirds of the foundation year-1 cohort made some contribution to the programme, either by lecture, bedside teaching or mock OSCE examination, which allows for a meaningful interpretation in the context of the perceived teaching ability of foundation doctors in general.

It is important to note that this study only attempts to assess perceived value of the teaching programme, no objective assessment of teaching impact has been made. There is also potential for considerable response bias, due to the manner in which the data were collected. Students are well aware that teaching sessions from those below the level of specialist registrar are compiled and delivered in their free time. There is undoubted risk that this may have a positive impact on scores given in the questionnaires. However, the same degree of response bias applies to each facet of teaching, allowing 
meaningful comparison of the differences in perceived value of different facets of the teaching.

We were not able to analyse all the facets of the teaching programme that we had intended thus limiting the number of conclusions that can be drawn. This was because the end-of-programme questionnaire data were invalidated by the duplication of responses. This duplication could have been avoided, while maintaining anonymity, by the use of unique identifiers for responders and this could be usefully incorporated into future research methodology in this area.

There are two main theories put forward to explain how someone of only limited extra experience may still serve as a useful learning resource.

One argument put forward in support of near-peer teaching is that of cognitive congruence. It is suggested that an individual with a similar knowledge base to the student will have a similar knowledge framework, providing an advantage in conveying that information when compared against someone with considerably greater knowledge and understanding (and therefore a more complex knowledge framework). ${ }^{17}$

It has also been suggested that near-peer teachers are able to communicate more effectively thus providing a better educational environment. ${ }^{8} 18-20$

This study has gone further than previous ones in the literature by analysing which facets of teaching and the teacher were preferred. The take home message from this was that the teachers scored highly in all areas. Unsurprisingly, the foundation and core trainees scored less well on knowledge and better on enthusiasm, interactivity and communication, adding some support to the theory that near-peer teaching provides an informal environment which is advantageous to learning. ${ }^{8} 18$ The students' perception of the teachers as enthusiastic and interactive may have facilitated more open discussion, aiding perceived learning even in our formal didactic sessions.

Given the availability of foundation and core trainees and the enthusiasm for teaching within the profession the authors feel that they are a resource that should be utilised by all medical schools in a formal programme. If to teach is to learn twice, then expanding the role of foundation and core trainees in undergraduate education would also serve as a valuable post-graduate education tool, and reduce consultants' undergraduate education commitments, ultimately providing more time for them to teach the teachers.

Acknowledgements Dr Sophie Tanner who was invaluable in helping to organise the 'mock' OSCE which was integral to the success of the programme.
Contributors GM and LG played equal roles in designing the study, delivering the programme, analysing data and both contributed to both the original draft and revisions.

Funding This research received no specific grant from any funding agency in the public, commercial or not-for-profit sectors.

Competing interests None.

Provenance and peer review Not commissioned; externally peer reviewed.

Data sharing statement Extra data can be accessed via the Dryad data repository at http://datadryad.org/ with the doi:10.5061/dryad.9rf90

\section{REFERENCES}

1. Bulte C, Betts A, Garner K, et al. Student teaching: views of student near-peer teachers and learners. Med Teach 2007;29:583-90.

2. Dandavino M, Snell L, Wiseman J. Why medical students should learn how to teach. Med Teach 2007;29:558-65.

3. General Medical Council. Good Medical PracticeG (Internet). 2009. http://www.gmc-uk.org/guidance/good_medical_practice.asp (accessed 19 Feb 2012).

4. Haist SA, Wilson JF, Fosson SE, et al. Are fourth-year medical students effective teachers of the physical examination to first-year medical students? J Gen Intern Med 1997;12:177-81.

5. Rodrigues J, Sengupta A, Mitchell A, et al. The Southeast Scotland Foundation Doctor Teaching Programmeme-is 'near-peer' teaching feasible, efficacious and sustainable on a regional scale? Med Teach 2009;31:e51-7.

6. Rashid MS, Sobowale O, Gore D. A near-peer teaching programme designed, developed and delivered exclusively by recent medical graduates for final year medical students sitting the final objective structured clinical examination (OSCE). BMC Med Educ 2011;11:11.

7. Kibble JD. A peer-led supplemental tutorial project for medical physiology: implementation in a large class. Adv Physiol Educ 2009;33:111-14.

8. Ten Cate O, Durning S. Peer teaching in medical education: twelve reasons to move from theory to practice. Med Teach 2007;29:591-9.

9. Batchelder AJ, Rodrigues CMC, Lin L-Y, et al. The role of students as teachers: four years' experience of a large-scale, peer-led programmeme. Med Teach 2010;32:547-51.

10. Alcamo AM, Davids AR, Way DP, et al. The impact of a peer-designed and -led USMLE step 1 review course: improvement in preparation and scores. Acad Med 2010;85(10 Suppl):S45-8.

11. Stanger-Hall KF, Lang S, Maas M. Facilitating learning in large lecture classes: testing the 'teaching team' approach to peer learning. CBE Life Sci Educ 2010;9:489-503.

12. Preszler RW. Replacing lecture with peer-led workshops improves student learning. CBE Life Sci Educ 2009;8:182-92.

13. Brazeau C, Boyd L, Crosson J. Changing an existing OSCE to a teaching tool: the making of a teaching OSCE. Acad Med2002;77:932

14. Colaco SM, Chou CL, Hauer KE. Near-peer teaching in a formative clinical skills examination. Med Educ 2006;40:1129-30.

15. Knobe $M$, Münker $R$, Sellei $R$, et al. Peer teaching: a randomised controlled trial using student-teachers to teach musculoskeletal ultrasound. Med Educ 2010;44:148-55.

16. Nestel D, Kidd J. Peer tutoring in patient-centred interviewing skills: experience of a project for first-year students. Med Teach 2003;25:398-403.

17. Tolsgaard MG, Gustafsson A, Rasmussen MB, et al. Student teachers can be as good as associate professors in teaching clinical skills. Med Teach 2007;29:553-7.

18. Lockspeiser TM, O'Sullivan P, Teherani A, et al. Understanding the experience of being taught by peers: the value of social and cognitive congruence. Adv Health Sci Educ Theory Pract 2008;13:361-72.

19. Evans DJR, Cuffe T. Near-peer teaching in anatomy: an approach for deeper learning. Anat Sci Educ 2009;2:227-33.

20. Leeper H, Chang E, Cotter G, et al. A student-designed and student-led sexual-history-taking module for second-year medical students. Teach Learn Med 2007;19:293-301. 